\title{
Efeitos psicossociais do distanciamento social durante as infecções por coronavírus: revisão integrativa
}

Psychosocial effects of social distancing during coronavirus infections: integrative review Efectos psicosociales del distanciamiento social durante las infecciones por coronavirus: revisión integradora

Daniel de Macêdo Rocha ${ }^{1}$ io https://orcid.org/0000-0003-1709-2143

Joyce Soares e Silva ${ }^{1}$ id https://orcid.org/ 0000-0001-6544-9632

Ingrid Moura de Abreu ${ }^{1}$ id https://orcid.org/0000-0003-1785-606x

Priscila Martins Mendes ${ }^{1}$ io hitps://orcid.org/0000-0001-8905-3931

Hilda Dandara Carvalho Santos Leite ${ }^{1}$ io https://orcid.org/0000-0002-8095-2874

Maria do Carmo Santos Ferreira ${ }^{1}$ io https://orcid.org/0000-0002-7180-9208 Como citar:
Rocha DM, Silva JS, Abreu IM, Mendes PM, eite HD, Ferreira MC. Efeitos psicossociais do distanciamento social durante as infeccões por coronavírus: revisão integrativa. Acta Paul Enferm. 2021;34:APE01141.

DOI http://dx.doi.org/10.37689/actaape/2021AR01141

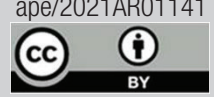

Isolamento social; Saúde Mental; Estresse psicológico; Infecções por coronavírus; Coronavírus

Keywords

Social isolation; Mental health; Stress, psychological; Coronavirus infections; Coronavirus

\section{Descriptores}

Aislamiento social; Salud mental; Estrés psicológico; Infecciones por coronavirus;

Coronavirus

Submetido 15 de Maio de 2020

Aceito

2 de Dezembro de 2020

\section{Autor correspondente}

Daniel de Macêdo Rocha

E-mail: daniel_m.rocha@outlook.com.br

\section{Resumo}

Objetivo: Analisar na literatura os efeitos psicossociais do isolamento e do distanciamento social adotados como estratégia para prevenção e controle das infecções por coronavírus.

Métodos: Revisão integrativa fundamentada no referencial teórico proposto por Whittemore e Knafl e executada em sete recursos informacionais: MEDLINE, SCOPUS, Embase, Web of Science, LILACS, IBECS e BDENF. A amostra foi constituída por 14 estudos primários, sem delimitação temporal ou de idioma e a análise foi realizada de forma descritiva. Utilizou-se a classificação do nível de evidência para caracterização das produções.

Resultados: As repercussões psicossociais decorrentes das medidas de isolamento e distanciamento social foram frequentes em diferentes países, indicando impactos negativos na saúde mental e na qualidade de vida. Os comprometimentos psicológicos foram expressos por instabilidades de humor, níveis elevados de ansiedade, situação de estresse, frustração, solidão, raiva e alteração de padrão de sono. Fatores associados à duração prolongada da medida, às possibilidades de contágio, às instabilidades econômicas, ao desconhecimento e às incertezas que permeiam a doença foram determinantes para o grau de sofrimento psíquico.

Conclusão: As medidas de distanciamento e isolamento social apesar de contribuírem para o controle epidemiológico em surtos, epidemias e pandemia por coronavírus, geraram comprometimentos na saúde mental da população, em que a duração da medida, as possibilidades de contágio, o medo e as instabilidades financeiras foram determinantes para o grau de sofrimento psíquico.

\section{Abstract}

Objective: To analyze the literature regarding the psychosocial effects of isolation and social distancing measures adopted as strategy for the prevention and control of coronavirus infections.

Methods: Integrative review of the literature based on the theoretical framework proposed by Whittemore and Knafl and conducted on seven databases: MEDLINE, SCOPUS, Embase, Web of Science, LILACS, IBECS and BDENF. The sample consisted of 14 primary studies, with no time or language restriction and the analysis was descriptive. The level of evidence classification was used to characterize the studies.

Results: Psychosocial repercussions of isolation and social distancing measures were frequent in different countries, with a negative impact on mental health and quality of life. Psychological impairments included mood instability, high levels of anxiety, stress, frustration, loneliness, anger and altered sleep patterns. Factors related to the prolonged duration of the measure, possibilities of contagion, economic instability, lack of knowledge and the uncertainties related to the disease were associated with psychological distress. 
Conclusion: Isolation and social distancing measures contribute to the epidemiological control of outbreaks, epidemics and pandemics of coronavirus; however, they compromise the population's mental health. The duration of the measure, the possibilities of contagion, fear and financial instability were determining factors for the degree of psychological distress.

\section{Resumen}

Objetivo: Analizar en la literatura los efectos psicosociales del aislamiento y del distanciamiento social adoptados como estrategia de prevención y control de las infecciones por coronavirus.

Métodos: Revisión integradora fundamentada en el marco referencial teórico propuesto por Whittemore y Knafl y ejecutada en siete recursos informativos: MEDLINE, SCOPUS, Embase, Web of Science, LILACS, IBECS y BDENF. La muestra estuvo compuesta por 14 estudios primarios, sin delimitación temporal ni de idioma, y el análisis se realizó de forma descriptiva. Se utilizó la clasificación del nivel de evidencia para la caracterización de las producciones.

Resultados: Las repercusiones psicosociales resultantes de las medidas de aislamiento y distanciamiento social fueron frecuentes en diferentes países e indicaron impactos negativos en la salud mental y en la calidad de vida. Las consecuencias psicológicas expresadas fueron humor inestable, niveles elevados de ansiedad, situación de estrés, frustración, soledad, enojo y cambios en el patrón de sueño. Factores asociados a la duración prolongada de la medida, a las posibilidades de contagio, a la inestabilidad económica, al desconocimiento y a la incertidumbre que provoca la enfermedad fueron determinantes para el nivel de sufrimiento psíquico.

Conclusión: Las medidas de distanciamiento y aislamiento social, a pesar de contribuir con el control epidemiológico en brotes, epidemias y pandemia por coronavirus, generan consecuencias en la salud mental de la población; y la duración prolongada de la medida, las posibilidades de contagio, el miedo y la inestabilidad económica fueron determinantes para el nivel de sufrimiento psíquico.

\section{Introdução}

Os últimos 30 anos foram marcados pela ascensão de doenças emergentes e reemergentes que representam ameaça à saúde pública mundial, em razão do seu potencial para disseminação global. Nesse contexto, nos anos de 2002 a 2010 surtos epidêmicos provocados por coronavírus e caracterizados por síndromes respiratórias acarretaram importante impacto epidemiológico, econômico, laboral, social e de saúde. ${ }^{(1,2)}$

Em 2019 uma nova pneumonia por coronavírus identificada na cidade de Wuhan, China foi referida pela Organização Mundial da Saúde (OMS) como COVID-19, tornando-se problema de alta magnitude ao ser reconhecida como pandemia e ao apresentar repercussóes na saúde mental, requerendo a restruturação do atendimento em diferentes contextos e níveis de atençăo. ${ }^{(2,3)}$

Descrita como infecção predominantemente humana, as estratégias de cuidados para prevenção e controle tornaram-se desafio constante em meio ao processo de globalização. Embora evidenciados os meios de propagação e características epidemiológicas, inúmeras lacunas são alvos de investigaçôes e envolvem consequências físicas, mentais, métodos diagnósticos e recursos terapêuticos. ${ }^{(4,5)}$

Apesar de ser considerada como infecção progressiva e com alto potencial de disseminação, a COVID-19 apresenta baixa patogenicidade, estan- do os indicadores de mortalidade expressos, em sua maioria, nas pessoas que apresentam deficiências ou diminuição da resposta imunológica, seja pelo processo de envelhecimento ou por condições pré-existentes, que os configuram como grupo de maior risco. ${ }^{(6)}$

Frente a isso, dentre os recursos referenciados na literatura e adotado por países de baixa, média e alta renda para garantir a dissoluçáo da curva epidêmica e evitar colapsos nos sistemas de saúde destacaram-se o isolamento e distanciamento social. Tratam-se de métodos eficazes para minimizar os indicadores de morbimortalidade mediante atraso da propagação viral resultante da permanência e restrição da população ao ambiente domiciliar. ${ }^{(7-10)}$

Em meio a esses recursos, efeitos psicossociais podem surgir ou se intensificar no ambiente domiciliar e repercutir severamente na saúde mental, no funcionamento e na estruturação familiar. Tratamse de repercussốes econômicas, sociais e psicológicas que podem refletir na perda da produtividade, na pior percepçáo do estado global de saúde e da qualidade de vida. Assim, diferentes fatores estressores decorrentes de instabilidades emocionais, da veiculação de informaçôes falsas e da perda de renda podem ser vivenciados e representar situaçáo de risco, requerendo medidas de suporte e gerenciamento de cuidados. ${ }^{(2,10)}$

Considerando as lacunas no conhecimento relacionados as disfunçóes na saúde mental e ao au- 
mento expressivo de comorbidades psicopatológicas durante a pandemia, assim como a necessidade de linhas integrais de cuidados, este estudo tem como objetivo analisar na literatura os efeitos psicossociais do isolamento e distanciamento social adotados como estratégia para prevenção e controle das infecções por coronavírus.

\section{Métodos}

Revisão integrativa fundamentada no referencial teórico proposto por Whittemore e Knafl e conduzida em seis etapas de investigação: elaboração da questão de pesquisa; busca e amostragem na literatura; definição das informaçóes a serem extraídas dos artigos selecionados; avaliação crítica das evidências incluídas; interpretação dos resultados; síntese do conhecimento e apresentação da revisão. ${ }^{(11)}$

A questão de pesquisa foi elaborada por meio do acrônimo PICo, definindo como problema o distanciamento e isolamento social, como fenômeno de interesse as repercussóes psicossociais e como contexto a infecção por coronavírus. ${ }^{(12)}$ Desse modo, está investigação foi conduzida pela seguinte questão: Quais os efeitos psicossociais do distanciamento e isolamento social adotado como estratégia de prevenção e controle das infecções por coronavírus?

O levantamento bibliográfico foi realizado em março e abril de 2020, mediante consulta nos recursos informacionais Medical Literature Analysis and Retrieval System on-line (MEDLINE via PubMed'), SCOPUS, Embase, Web of Science ${ }^{\mathrm{TM}}$, SCOPUS, Literatura Latino-Americana e do Caribe em Ciências da Saúde (LILACS), Índice Bibliográfico Espanhol de Ciências da Saúde (IBECS) e Banco de Dados em Enfermagem (BDENF) via Biblioteca Virtual em Saúde.

Para operacionalização da busca foram selecionados descritores controlados e não controlados foram extraídos dos vocabulários Descritores em Ciências da Saúde (DeCS) e Medical Subject Headings (MESH). O quadro 1 descreve a estratégia gerada em cada recurso informacional consultado a partir da combinação dos termos com os operadores booleanos $O R$ e $A N D$.
Quadro 1. Expressão de busca gerada nos recursos informacionais investigados

\begin{tabular}{|c|c|}
\hline Base de dados & Expressão de busca \\
\hline MEDLINE & 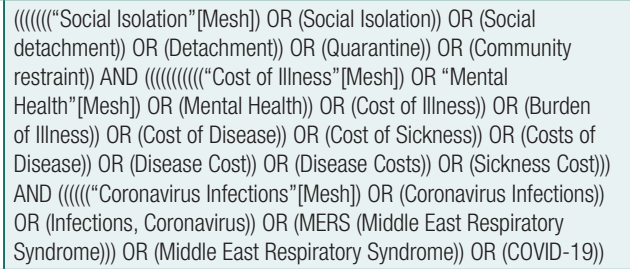 \\
\hline SCOPUS & $\begin{array}{l}\text { (TITLE-ABS-KEY ( social AND isolation) OR TITLE-ABS-KEY ( } \\
\text { social AND detachment) OR TITLE-ABS-KEY (detachment) OR } \\
\text { TITLE-ABS-KEY ( quarantine) OR TITLE-ABS-KEY ( community AND } \\
\text { restraint) ) AND ( TITLE-ABS-KEY (cost AND of AND illness ) OR } \\
\text { TITLE-ABS-KEY ( mental AND health) OR TITLE-ABS-KEY (burden } \\
\text { AND of AND illness) OR TITLE-ABS-KEY ( cost AND of AND } \\
\text { disease) OR TITLE-ABS-KEY (cost AND of AND sickness) OR } \\
\text { TITLE-ABS-KEY (costs AND of AND disease) OR TITLE-ABS-KEY } \\
\text { ( disease AND cost) OR TITLE-ABS-KEY ( disease AND costs) } \\
\text { OR TITLE-ABS-KEY ( sickness AND cost)) AND (TITLE-ABS-KEY ( } \\
\text { coronavirus AND infections) OR TITLE-ABS-KEY (mers) OR TITLE- } \\
\text { ABS-KEY ( middle AND east AND respiratory AND syndrome) OR } \\
\text { TITLE-ABS-KEY (covid-19)) }\end{array}$ \\
\hline Embase & $\begin{array}{l}\text { (social AND isolation OR (social AND detachment) OR detachment } \\
\text { OR quarantine OR (community AND restraint)) AND (cost AND of AND } \\
\text { illness OR (mental AND health) OR (burden AND of AND illness) OR (cost } \\
\text { AND of AND disease) OR (cost AND of AND sickness) OR (costs AND } \\
\text { of AND disease) OR (disease AND cost) OR (disease AND costs) OR } \\
\text { (sickness AND cost)) AND (coronavirus AND infections OR (infections, } \\
\text { AND coronavirus) OR (mers AND middle AND east AND respiratory AND } \\
\text { syndrome) OR (middle AND east AND respiratory AND syndrome) OR } \\
\text { 'covid 19') }\end{array}$ \\
\hline Web of Science & $\begin{array}{l}\text { TS=((Social Isolation) OR (Social detachment) OR (Detachment) OR } \\
\text { (Quarantine) OR (Community restraint)) AND TS=((Cost of Illness) OR } \\
\text { (Mental Health) OR (Burden of Illness) OR (Cost of Disease) OR (Cost of } \\
\text { Sickness) OR (Costs of Disease) OR (Disease Cost) OR (Disease Costs) } \\
\text { OR (Sickness Cost)) AND TS=((Coronavirus Infections) OR (Infections, } \\
\text { Coronavirus) OR (MERS (Middle East Respiratory Syndrome)) OR } \\
\text { (Middle East Respiratory Syndrome) OR (COVID-19)) }\end{array}$ \\
\hline $\begin{array}{l}\text { LILACS, BDENF e } \\
\text { IBECS }\end{array}$ & $\begin{array}{l}\text { (mh:(Isolamento Social OR Distanciamento social OR Distanciamento } \\
\text { OR Quarentena OR Contenção comunitária)) AND (mh:(Efeitos } \\
\text { Psicossociais da Doença OR Saúde Mental OR Carga da Doença } \\
\text { OR Custo da Doença OR Fardo da Doença OR Ônus da Doença )) } \\
\text { AND (mh:(Infecçõs por Coronavirus OR COVID-19 OR Doença por } \\
\text { Coronavírus 2019-nCoV OR Infecção pelo Coronavírus 2019-nCoV OR } \\
\text { Infecções por Coronavírus)) }\end{array}$ \\
\hline
\end{tabular}

Foram incluídos estudos primários que investigaram as relações entre as repercussóes psicossociais, isolamento e o distanciamento social utilizado para prevenção e controle de infecçóes associadas ao coronavírus ou como medida de suporte para outras síndromes respiratórias graves, sem delimitação temporal ou de idioma. Estudos duplicados, editoriais, teses, dissertações e revisão foram excluídos.

A busca e a inclusão foram realizadas por dois revisores, de forma independente e com índice de concordância de resultados superior a $80 \%$. As discordâncias $(n=62)$ foram gerenciadas por um terceiro revisor que emitiu um parecer quanto à inclusão.

A busca totalizou 3.109 produçóes e após a aplicação dos critérios de elegibilidade, obteve-se a amostra de 14 artigos. Ressalta-se que não foram 
recuperados estudos nos recursos informacionais LILACS, IBECS e BDENF. Dessa forma, não foi considerada para fins de coleta de dados. O percurso realizado para identificação, seleção, elegibilidade, inclusão e amostra seguiu as recomendações do Preferred Reporting Items for Systematic Reviews and Meta-Analyses (PRISMA), conforme apresentado na figura 1. ${ }^{(13)}$

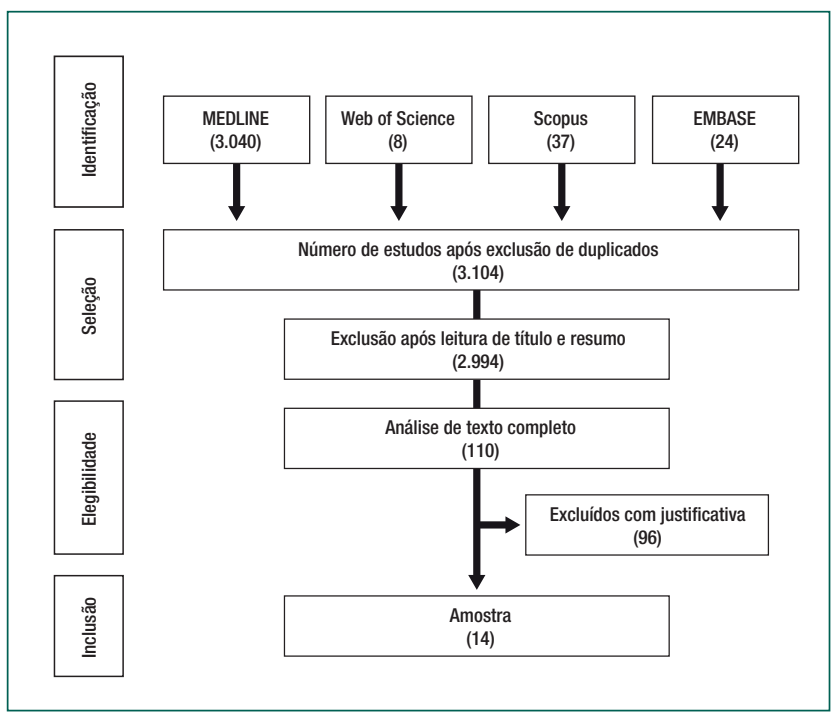

Fonte: Moher D, Liberati A, Tetzlaff J, Altman DG; PRISMA Group. Preferred reporting items for systematic reviews and meta-analyses: the PRISMA statement. PLoS Med. 2009;6(7):e1000097. ${ }^{(13)}$

Figura 1. Percurso de seleção dos estudos primários

Para extração dos dados adaptou-se um instrumento validado, sendo consideradas variáveis de identificação do estudo (autor principal, objetivo, local do estudo e ano de publicaçáo), aspectos metodológicos (delineamento e nível de evidência), principais resultados e conclusôes. ${ }^{(14)}$

As recomendaçóes propostas pelo Oxford Centre for Evidence-based Medicine foram utilizadas para classificação do nível de evidência, considerando: $1 \mathrm{~A}$ - revisão sistemática de ensaios clínicos controlados randomizados; 1B - ensaio clínico controlado randomizado com intervalo de confiança estreito; $1 \mathrm{C}$ - resultados terapêuticos do tipo "tudo ou nada"; $2 \mathrm{~A}$ - revisão sistemática de estudos de coorte; $2 \mathrm{~B}$ - estudo de coorte (incluindo ensaio clínico randomizado de menor qualidade); 2C - observação de resultados terapêuticos ou estudos ecológicos; $3 \mathrm{~A}$ - revisão sistemática de estudos caso-controle; 3B - estudo caso-controle; 4 - relato de casos (incluindo coorte ou caso-controle de menor qualidade); 5 opinião de especialistas. ${ }^{(15)}$

A análise e síntese dos resultados foram realizadas de forma descritiva e apresentadas em quadro conforme variáveis de interesse para este estudo. Para tanto, as evidências foram agrupadas em duas categorias: Efeitos psicossociais do isolamento e distanciamento social; e, Fatores associados aos efeitos psicossociais do isolamento e distanciamento social.

\section{Resultados}

Dentre os estudos incluídos, verificaram-se que os resultados, em sua totalidade, foram publicados em idioma inglês e desenvolvidos em diferentes países como China, Coreia do Sul e Canadá que vivenciaram surtos, epidemias e pandemias relacionadas aos coronavírus. Predominaram produçóes realizadas em 2020 (50\%) e que apresentaram delineamentos observacionais analíticas, nível de evidência $2 \mathrm{C}$ $(85,71 \%)$.

Em relação às repercussóes psicossociais vivenciadas ou intensificadas durante o isolamento e distanciamento social durantes surtos de coronavírus, foram realidades frequentes entre os segmentos populacionais de diferentes países e expressos, na maioria das vezes, por instabilidades de humor e sintomas psicossomáticos de ansiedade. Outros eventos vivenciados que comprometeram a saúde mental da população estiveram associados ao medo, situaçôes de estresse, frustração, solidão, raiva e alteração de padrão de sono.

Ainda, foi evidenciado que diferentes fatores representam determinantes para o grau de comprometimento da saúde mental, estando relacionados à duração prolongada da medida, ao desconhecimento e incertezas que permeiam a doença, as possibilidades de contágio, e aos impactos financeiros que podem levar a redução de renda familiar, restriçáo alimentar e instabilidades econômicas. Destaca-se que a presença de sintomatologias que cursaram com o quadro clínico da infecção também constituiu um dos preditores para o desenvolvimento de reaçôes emocionais. 
Quadro 2. Síntese dos estudos incluídos na revisão $(n=14)$

\begin{tabular}{|c|c|c|c|}
\hline $\begin{array}{l}\text { Autor principal, } \\
\text { ano e local }\end{array}$ & Objetivo & $\begin{array}{l}\text { Delineamento } \\
\text { e NE }\end{array}$ & Efeitos psicossociais e fatores associados \\
\hline $\begin{array}{l}\text { Xiao } \mathrm{H}^{(16)} \\
2020 \\
\text { China }\end{array}$ & \begin{tabular}{|l|} 
Investigar os efeitos do capital social na qualidade \\
do sono e os mecanismos envolvidos em pessoas \\
que se isolaram durante a epidemia COVID-19. \\
\end{tabular} & $\begin{array}{l}\text { Quantitativo } \\
2 \mathrm{C}\end{array}$ & $\begin{array}{l}\text { Ansiedade, estresse e alteração do padrão de sono } \\
\text { Impacto financeiro. }\end{array}$ \\
\hline $\begin{array}{l}\text { Wang } Y^{(17)} \\
2020 \\
\text { China }\end{array}$ & $\begin{array}{l}\text { Avaliar os estados psicológicos e seus fatores } \\
\text { relacionados durante } 0 \text { surto do COVID-19 }\end{array}$ & $\begin{array}{l}\text { Coorte } \\
2 B\end{array}$ & $\begin{array}{l}\text { Ansiedade e depressão } \\
\text { Medo do contágio }\end{array}$ \\
\hline $\begin{array}{l}\text { Roy } D^{(18)} \\
2020 \\
\text { Índia }\end{array}$ & $\begin{array}{l}\text { Avaliar o conhecimento, a atitude, a ansiedade } \\
\text { e as necessidades de saúde mental durante a } \\
\text { pandemia de coronavirus. }\end{array}$ & $\begin{array}{l}\text { Observacional } \\
2 \mathrm{C}\end{array}$ & $\begin{array}{l}\text { Ansiedade, insônia e paranoias } \\
\text { Pouco conhecimento sobre a doença, preocupação e medo frente às informações divulgadas em mídias } \\
\text { sociais. }\end{array}$ \\
\hline $\begin{array}{l}\text { Lei } L^{(19)} \\
2020 \\
\text { China }\end{array}$ & $\begin{array}{l}\text { Avaliar a prevalência e fatores associados à } \\
\text { ansiedade e depressão durante quarentena do } \\
\text { COVID-19 }\end{array}$ & $\begin{array}{l}\text { Observacional } \\
2 \mathrm{C}\end{array}$ & $\begin{array}{l}\text { Elevado nível de ansiedade e depressão } \\
\text { Medo de contágio, baixa renda e escolaridade, falta de apoio psicológico e preocupação em ser infectado. }\end{array}$ \\
\hline $\begin{array}{l}\text { Yuan } S^{(20)} \\
2020 \\
\text { China }\end{array}$ & $\begin{array}{l}\text { Comparar o estado emocional, respostas } \\
\text { somáticas, qualidade do sono e comportamento } \\
\text { de pessoas durante isolamento social }\end{array}$ & $\begin{array}{l}\text { Observacional } \\
2 \mathrm{C}\end{array}$ & Ansiedade, má qualidade do sono e estresse. \\
\hline $\begin{array}{l}\text { Koolaee AK(21) } \\
2020 \\
\text { Iran }\end{array}$ & $\begin{array}{l}\text { Analisar as experiências psicológicas de } \\
\text { estudantes universitários em quarentena } \\
\text { doméstica. }\end{array}$ & $\begin{array}{l}\text { Qualitativa } \\
\text { 2C }\end{array}$ & $\begin{array}{l}\text { Comportamentos obsessivo-compulsivos e emoç̃oses negativas. } \\
\text { Medo de contaminação, preocupações financeiras, sociais e com a saúde familiar. }\end{array}$ \\
\hline $\begin{array}{l}\text { Lee SM(22) } \\
2018 \\
\text { Coreia do Sul }\end{array}$ & \begin{tabular}{|l} 
Avaliar o estresse imediato e 0 impacto \\
psicológico vivenciado na quarentena durante a \\
Síndrome Respiratória do Oriente Médio (MERS).
\end{tabular} & $\begin{array}{l}\text { Quantitativo } \\
2 \mathrm{C}\end{array}$ & $\begin{array}{l}\text { Sintomas de transtorno de estresse pós-traumático. } \\
\text { Medo de contaminação e alta mortalidade. }\end{array}$ \\
\hline $\begin{array}{l}\text { Kim } \mathrm{HC}^{(23)} \\
2018 \\
\text { Coreia do Sul }\end{array}$ & $\begin{array}{l}\text { Avaliar as complicações psiquiátricas ou os } \\
\text { fatores de risco para depressão em pacientes com } \\
\text { MERS em quarentena. }\end{array}$ & $\begin{array}{l}\text { Observacional } \\
2 \mathrm{C}\end{array}$ & $\begin{array}{l}\text { Depressão e outros sintomas de ordens psiquiátricas. } \\
\text { Falta de conhecimento sobre a doença e perdas financeiras. }\end{array}$ \\
\hline $\begin{array}{l}\text { Jeong } \mathrm{H}^{(24)} \\
2016 \\
\text { Coreia do Sul }\end{array}$ & \begin{tabular}{|l|} 
Examinar a prevalência de sintomas de ansiedade \\
e raiva em pessoas isoladas durante a epidemia \\
de MERS
\end{tabular} & $\begin{array}{l}\text { Quantitativo } \\
2 \mathrm{C}\end{array}$ & $\begin{array}{l}\text { Ansiedade e raiva } \\
\text { Isolamento prolongado, suprimentos alimentares insuficientes, perdas financeiras e histórico de doenças } \\
\text { psiquiátricas. }\end{array}$ \\
\hline $\begin{array}{l}\text { Yoon MK'(25) } \\
2016 \\
\text { Coreia do Sul }\end{array}$ & $\begin{array}{l}\text { Relatar o caso de intervenção proativa baseada na } \\
\text { comunidade na Coréia do Sul. }\end{array}$ & $\begin{array}{l}\text { Relato de caso } \\
4\end{array}$ & $\begin{array}{l}\text { Dificuldades psicológicas e instabilidades emocionais. } \\
\text { Quarentena prolongada }\end{array}$ \\
\hline $\begin{array}{l}\text { Mihashi M(26) } \\
2009 \\
\text { Japão }\end{array}$ & $\begin{array}{l}\text { Investigar estratégias para amplo isolamento de } \\
\text { massa durante surtos de doenças infecciosas. }\end{array}$ & $\begin{array}{l}\text { Quantitativo } \\
2 \mathrm{C}\end{array}$ & $\begin{array}{l}\text { Instabilidades emocionais } \\
\text { Redução de renda, restrição alimentar e do convívio social e apresentar sintomas clínicos. }\end{array}$ \\
\hline $\begin{array}{l}\text { Hawryluck L27) } \\
2004 \\
\text { Canadá }\end{array}$ & $\begin{array}{l}\text { Entender as efeito psicológico em pessoas em } \\
\text { quarentena durante os recentes surtos de SARS. }\end{array}$ & $\begin{array}{l}\text { Quantitativo } \\
2 \mathrm{C}\end{array}$ & $\begin{array}{l}\text { Sintomas de transtorno de estresse pós-traumático. } \\
\text { Duração longa da medida, baixo conhecimento da doença e exposição direta a alguém com diagnóstico. }\end{array}$ \\
\hline $\begin{array}{l}\text { Robertson } E^{(28)} \\
2004 \\
\text { Canadá }\end{array}$ & $\begin{array}{l}\text { Examinar os efeitos psicossociais dos profissionais } \\
\text { de saúde em quarentena devido à SARS. }\end{array}$ & $\begin{array}{l}\text { Qualitativo } \\
\text { 2C }\end{array}$ & $\begin{array}{l}\text { Medo e frustração } \\
\text { Perda de intimidade e contato social, conflito decorrente de papéis simultâneos, medo em infectar } \\
\text { familiares e amigos vulneráveis. }\end{array}$ \\
\hline $\begin{array}{l}\text { Maunder } \mathrm{R}^{(22)} \\
2003 \\
\text { Canadá }\end{array}$ & $\begin{array}{l}\text { Descrever o impacto psicológico e ocupacional do } \\
\text { surto de SARS em hospital de grande porte nas } \\
\text { primeiras } 4 \text { semanas. }\end{array}$ & $\begin{array}{l}\text { Observacional } \\
2 \mathrm{C}\end{array}$ & $\begin{array}{l}\text { Ansiedade, insônia } \\
\text { medo, solidão, tédio e raiva. } \\
\text { Preocupação de contágio nos familiares e amigos, presença de sintomatologias clínicas, incerteza e estigmatização. }\end{array}$ \\
\hline
\end{tabular}

A síntese e a distribuição das produçóes incluídas estáo apresentadas no Quadro 2, conforme objetivo do estudo, ano e local de publicação, delineamento adotado e Nível de Evidência (NE), efeitos psicossociais decorrentes das medidas de isolamento e distanciamento social, assim como seus fatores associados.

\section{Discussão}

Nesta revisão, a concentração de estudos desenvolvidos no ano 2020 evidencia o crescente interesse dos pesquisadores em buscar alternativas para gerenciar as repercussóes psicossociais decorrentes da COVID-19, que ao ser declarado estado de pande- mia desestruturou contextos sociais, econômicos e de saúde em todo o mundo.

\section{Efeitos psicossociais do isolamento $\mathrm{e}$ distanciamento social}

$\mathrm{O}$ curso e a gravidade de um surto, epidemia ou pandemia relacionada às infecçóes por coronavírus exigiram das organizaçóes de saúde que intervençóes efetivas, sustentáveis e baseadas em evidências fossem implementadas como mecanismo primordial para conter a velocidade de transmissão, reduzir a sobrecarga social e os indicadores de morbimortalidade. ${ }^{(8,9)}$

Apesar de acarretar alteraçóes nos padróes de vida da população mundial, impactando na dinâmica econômica, política, cultural e psicossocial, as respostas governamentais dirigiram-se, sobretudo, 
à propagação de estratégias para distanciamento e isolamento social como evento promissor para controle epidemiológico da doença. ${ }^{(22,23)}$

Nesse sentido, o controle do coronavírus constitui prioridade para as redes de atenção à saúde. Permanece em segundo plano a atenção ao comprometimento psicológico de pessoas com transtornos psiquiátricos e àqueles com desordens mentais oriundas do isolamento e distanciamento social provocadas pela pandemia. ${ }^{(19)}$

Esta categoria evidencia que repercussóes psicossociais são frequentes em pessoas submetidas às medidas de isolamento e distanciamento social, que se configuram como condição determinante para os comprometimentos vivenciados, estando, na maioria dos estudos, associadas ao desenvolvimento ou a intensificação de sintomas de ansiedade e depressão. ${ }^{(16-20,22,24,29)}$

Consideradas as maiores causas de sofrimento emocional e diminuição da qualidade de vida, os sintomas de ansiedade representam as alteraçóes mais incidentes na população geral, constituindo resposta adaptativa do organismo em meio a um sinal de perigo ou ameaça, sendo expressa por condiçóes fisiológicas, comportamentais e cognitivas, e considerara patológica quando o nível de ativação ou duração é desproporcional à situação vivenciada. ${ }^{(18,19)}$

No transtorno depressivo o estado de perturbação mental manifestado por tristeza, perda de interesse e prazer, sentimento de culpa, baixa autoestima e perturbações do sono, pode representar condição de maior risco quando relacionado pelo comportamento suicida. Apesar de apresentar caráter evitável, ainda se configura como fenômeno universal, complexo, multifacetado, reconhecido, previsível e com elevados indicadores de mortalidade. ${ }^{(23,30)}$

Outros eventos verificados nas populaçóes estudadas envolveram estresse, raiva, paranoias e comportamentos compulsivos caracterizados por pensamentos, impulsos ou atos mentais recorrentes, em especial, relacionados a medidas de higiene. Esses eventos são reconhecidos pelo seu curso crônico, assim como pela interferência no ambiente familiar e diminuição da autoestima do bem-estar subjetivo. ${ }^{(16,20,21,24,29)}$

$\mathrm{Na}$ população geral, a prevalência de transtornos mentais e comportamentais é elevada, exigindo a necessidade de reavaliaçôes clínicas constatastes para manutenção terapêutica. Entretanto, o atendimento ambulatorial, modalidade de assistência especializada, encontra-se suspensa diante das medidas de quarentena, dificultando o acesso aos sistemas de saúde e contribuindo para recorrência de crises, reaçóes emocionais, situaçóes de conflitos e de violência. ${ }^{(25,26)}$

Outro estudo mostrou que os profissionais da saúde também desenvolveram comorbidades psicopatológicas quando submetidos a estratégias de isolamento em decorrência do adoecimento no ambiente laboral, sendo frequente sintomas de estresse pós-traumático, em que os altos níveis de ansiedade apresentaram-se durante e após o surto, exigindo intervenção psiquiátrica imediata e contínua. ${ }^{(22,28)}$

Assim, considera-se que as repercussóes do distanciamento e isolamento social prevalecem na população geral, podendo ocasionar impactos psicossociais e comprometimentos na qualidade de vida em diferentes contextos, apontando a necessidade de estratégias de cuidados e do direcionamento de políticas públicas que busquem a integralidade de cuidado e que valorizem a manutenção e preservação das funções psíquicas. ${ }^{(23)}$

\section{Fatores associados aos efeitos psicossociais do isolamento e distanciamento social}

De acordo com a análise realizada os fatores associados aos efeitos psicossociais decorrente do isolamento e distanciamento social, resumiram-se em: impacto e perdas financeiras, necessidade de restrição alimentar, aspectos relacionados a infecção como falta de conhecimento, medo do contágio de amigos e familiares, preocupação em meio aos relatórios divulgados em mídias sociais, apresentar histórico de doenças psiquiátricas.

O impacto financeiro foi vivenciado em diferentes países, evidenciando que durante as infecçóes por coronavírus os comprometimentos econômicos tornam-se problemas frequentes capazes de interferir no funcionamento familiar e nos níveis de saúde mental. ${ }^{(16)}$ Com fechamento dos serviços não essenciais como o comércio, restaurantes e construção civil, as pessoas precisaram interromper suas atividades laborais sem planejamento prévio ou reservas econômicas, resultando na perda financeira e sendo 
considerada importante fator de risco para o desenvolvimento de sintomas psicológicos. ${ }^{(26,31)}$

Estudo realizado durante a pandemia por COVID-19, no sudeste da China, também mostrou que a baixa renda familiar foi determinante dos níveis de sofrimento psíquico, uma vez que a ausência do trabalho acarretou riscos econômicos, necessitando de suporte governamental para o seu enfrentamento. ${ }^{(19)}$ Outras pesquisas revelaram que ao final da crise provocada pelas coronaviroses, o retorno à normalidade demorou meses, problemas socioeconômicos ficaram evidentes, houve aumento dos índices de desemprego e de trabalhadores autônomos. ${ }^{(23,24)}$

No Brasil, as medidas para auxílio financeiro e liberação de fundos de garantia, ambos em caráter emergencial, foram adotadas como suporte durante a pandemia de COVID-19 para combate não só da crise econômica, mas dos riscos psicológicos que a instabilidade financeira pode acarretar.

$\mathrm{O}$ medo do contágio também configurou fator associado aos efeitos psicossociais em meio as medidas de isolamento, ${ }^{(17)}$ uma vez que as preocupaçóes quanto as possibilidades de estarem infectadas ou de contaminação de familiares e amigos representaram condiçóes geradoras de morbidade. Além disso, as instabilidades emocionais decorrentes de sentimentos de raiva e revolta estiveram relacionadas à falta de coordenação, transparência e incongruências fornecidas pelo Estado e veiculado em massa por mídias socais quanto as medidas de segurança, indicadores de mortalidade e poder de disseminaçáo viral. ${ }^{(26-29)}$

O contexto político, econômico, social e de saúde que o Brasil vivencia é complexo e pode ser reflexo das grandes discussóes em mídias, assim como do seu potencial para disseminação de notícias falsas e sem comprovaçôes científicas. Nesse sentido as definiçōes de fake news se reestruturam para expressar, na maioria das vezes, conceitos errôneos causados por conteúdos precipitados, rumores, difamação, falta de autenticidade e propósito de enganar. ${ }^{(32)}$ Assim, as mídias podem constituir, neste estudo, fator associado à redução da saúde mental, podendo elevar sintomas de ansiedade, medo e pânico por extremismo. ${ }^{(18,19,23,27)}$

O histórico de enfermidade psiquiátrica também foi determinante para a saúde mental, uma vez que a predisposição para situaçóes de instabilidades emocionais pode intensificar situaçóes de crises e predizer estados de risco. As recomendaçóes de permanência em quarentena não incluem, em seus planejamentos, orientaçóes voltadas para o bem estar psicológico, que somado ao constrangimento por apresentar desordens psicológicas e a baixa adesão ao tratamento comum nessa população pode elevar os indicadores de sofrimento psíquico..$^{(19,20)}$

Estudo publicado em 2016 revelou que em outras epidemias por coronaviroses, houveram casos de pessoas, infectadas e em processo de recuperação, que demonstraram instabilidade emocional resultante da experiência de luto. Assim, os familiares de pessoas mortas por essas doenças apresentaram sérios impactos psicológicos após a perda repentina, reforçando a importância da preparação dos serviços de saúde mental, com acessibilidade adequada e sistemas eficazes para reconhecimento precoce de situaçôes de risco assim como para gerenciamento adequado. ${ }^{(25)}$

Em continuação acerca da discussão dos fatores associados aos efeitos psicossociais, a restrição alimentar ou recursos básicos corresponderam a um destes, podendo se estender também sobre medicamentos e produtos de limpeza que interfere no conforto e na qualidade de vida. ${ }^{(24)}$ Frente a isso, uma cultura de solidariedade social foi desenvolvida e fortalecida em diferentes países, sendo adotada como estratégia para atender as necessidades básicas da população e minimizar as repercussóes que o distanciamento e isolamento acarretam.

Ainda, a literatura destaca que os sentimentos de medo, as incertezas quanto a duração do distanciamento social e as consequências econômicas, políticas e de saúde, podem colocar as pessoas com sofrimento mental em situação de risco para contaminação pelo novo coronavírus, uma vez que sinais e sintomas psicossomáticos decorrentes dos níveis de ansiedade e pânico são comuns, elevando a busca por serviços emergenciais.

A limitação do estudo refere-se à prevalência de estudos com delineamentos observacionais, nível de evidência $2 \mathrm{C}$, por não estabelecer relaçôes de causa e efeito entre as variáveis de investigação. Entretanto, esse método representa recurso funda- 
mental para a prática clínica por demandar baixo custo e tempo para realização, assim como por favorecer a compreensão de fenômenos em investigação, sendo constantemente utilizados para indicar prognósticos, fatores de riscos e de proteção à saúde. ${ }^{(33)}$

\section{Conclusão}

O distanciamento e isolamento social adotado como medida para controle epidemiológico em surtos, epidemia e pandemia por coronavírus, impactou negativamente na saúde mental da população, predominando instabilidades de humor e sintomas psicossomáticos de ansiedade. Fatores associados à duração da medida, possibilidades de contágio, medo, instabilidades financeiras e restrição alimentar foram determinantes para maiores comprometimentos. Considera-se que estudos dessa natureza podem favorecer a compreensáo real dos impactos físicos, epidemiológicos, emocionais e financeiros que as infecçóes por coronavírus acarretam, despertando nos gestores, nos líderes de saúde e na prática assistencial de enfermagem a necessidade de políticas públicas e estratégias de cuidados voltadas para qualidade de vida, prevenção e gerenciamento adequado de estados de risco.

\section{Referências}

1. Ashton J. The pandemic of coronavirus: tackling the latest plague. J R Soc Med. 2020;113(3):123-4.

2. Farias HS. The advancement of Covid-19 and social isolation as a strategy to reduce vulnerability. Rev Bras Geog Econom. 2020, $4(17): 1-12$.

3. Farooq HZ, Davies E, Ahmad S, Machin N, Hesketh L, Guiver M et al. Middle East respiratory syndrome coronavirus (MERS-CoV) Surveillance and testing in North England from 2012 to 2019. Int J Infect Dis. 2020; 93:237-44.

4. Lai CC, Shih TP, Ko WC, Tang HJ, Hsueh PR. Severe acute respiratory syndrome coronavirus 2 (SARS-CoV-2) and coronavirus disease-2019 (COVID-19): the epidemic and the challenges. Int J Antimicrob Agents. 2020;55(3):105924.

5. Layne SP, Hyman JM, Morens DM, Taubenberger JK. New coronavirus outbreak: Framing questions for pandemic prevention. Sci Transl Med. 2020;12(534):eabb1469

6. Cui J, Li F, Shi ZL. Origin and evolution of pathogenic coronaviruses. Nat Rev Microbiol. 2019;17(3):181-92.
7. Tesarik J. After corona: there is life after the pandemic. Reprod Biomed Online. 2020;40(6):760-762.

8. Aranha FG. Novo coronavírus: o melhor cuidado é ficar isolado [editorial]. Arq Catarin Med. 2020; 49(1):1.

9. Hick JL, Biddinger PD. Novel Coronavirus and Old Lessons Preparing the Health System for the Pandemic. N Engl J Med. 2020;382(20):e55.

10. Khan S, Sidddique R, Ali A, Xue M, Nabi G. Novel coronavirus, poor quarantine, and the risk of pandemic. J Hosp Infect. 2020; 104(4):449-450.

11. Whittemore R, Knafl K. The integrative review: updated methodology. J Adv Nurs. 2005;52(5):546-53.

12. Lockwood C, Porrit K, Munn Z, Rittenmeyer L, Salmond S, Bjerrum $\mathrm{M}$, et al. Chapter 2: Systematic reviews of qualitative evidence. In: Aromataris E, Munn Z, editors. Australia: Joanna Briggs Institute; 2017.

13. Moher D, Liberati A, Tetzlaff J, Altman DG; PRISMA Group. Preferred reporting items for systematic reviews and meta-analyses: the PRISMA statement. PLoS Med. 2009;6(7):e1000097.

14. Marziale MH. Instrumento para recolección de datos revisión integrativa. 2015. Available from: http://gruposdepesquisa.eerp.usp. br/sites/redenso/wp-content/uploads/sites/9/2019/09/Instrumiento_ revision_litetarura_RedENS0_2015.pdf. Spanish.

15. Centre for Evidence Based Medicine (CEBM). Oxford Centre for Evidence-Based Medicine: Levels of Evidence (March 2009). Oxford: University of Oxford; c2009. [cited 2020 Nov 20]. Available from: https://www.cebm.ox.ac.uk/resources/levels-of-evidence/ oxford-centre-for-evidence-based-medicine-levels-of-evidencemarch-2009

16. Xiao H, Zhang Y, Kong D, Li S, Yang N. Social capital and sleep quality in individuals who self-isolated for 14 days during the coronavirus disease 2019 (COVID-19) Outbreak in January 2020 in China. Med Sci Monit. 2020;26:e923921.

17. Wang Y, Di Y, Ye J, Wei W. Study on the public psychological states and its related factors during the outbreak of coronavirus disease 2019 (COVID-19) in some regions of China. Psychol Health Med. 2020;30:1-10.

18. Roy D, Tripathy S, Kar SK, Sharma N, Verma SK, Kaushal V. Study of knowledge, attitude, anxiety \& perceived mental healthcare need in Indian population during COVID-19 pandemic. Asian J Psychiatry; 2020; 51:102083.

19. Lei L, Huang X, Zhang S, Yang J, Yang L, Xu M. Comparison of Prevalence and Associated Factors of Anxiety and Depression Among People Affected by versus People Unaffected by Quarantine During the COVID-19 Epidemic in Southwestern China. Med Sci Monit. 2020;26:e924609.

20. Yuan S, Liao Z, Huang H, Jiang B, Zhang X, Wang Y, et al. Comparison of the indicators of psychological stress in the population of hubei province and non-endemic provinces in china during two weeks during the coronavirus disease 2019 (COVID-19) outbreak in February 2020. Med Sci Monit. 2020;26:e923767.

21. Koolaee AK. Living in home quarantine: analyzing psychological experiences of college students during Covid-19 pandemic. J Military Med. 2020;22(2):130-8.

22. Lee SM, Kang WS, Cho AR, Kim T, Park JK. Psychological impact of the 2015 MERS outbreak on hospital workers and quarantined hemodialysis patients. Compr Psychiatry. 2018;87:123-7. 
23. Kim HC, Yoo SY, Lee BH, Lee SH, Shin HS. Psychiatric findings in suspected and confirmed middle east respiratory syndrome patients quarantined in hospital: a retrospective chart analysis. Psychiatry Investig. 2018;15(4):355-60.

24. Jeong H, Yim HW, Song YJ, Ki M, Min JA, Cho J, et al. Mental health status of people isolated due to Middle East Respiratory Syndrome. Epidemiol Health. 2016;38:e2016048.

25. Yoon MK, Kim SY, Ko HS, Lee MS. System effectiveness of detection, brief intervention and refer to treatment for the people with posttraumatic emotional distress by MERS: a case report of communitybased proactive intervention in South Korea. Int J Ment Health Syst. 2016;10(1):51.

26. Mihashi M, Otsubo Y, Yinjuan X, Nagatomi K, Hoshiko M, Ishitake T. Predictive factors of psychological disorder development during recovery following SARS outbreak. Health Psychol. 2009;28(1):91-100.

27. Hawryluck L, Gold WL, Robinson S, Pogorski S, Galea S, Styra R. SARS control and psychological effects of quarantine, Toronto, Canada. Emerg Infect Dis. 2004;10(7):1206-12.
28. Robertson E, Hershenfield K, Grace SL, Stewart DE. The psychosocial effects of being quarantined following exposure to SARS: a qualitative study of Toronto health care workers. Can J Psychiatry. 2004;49(6):403-7.

29. Maunder R, Hunter J, Vincent L, Bennett J, Peladeau N, Leszcz M, et al. The immediate psychological and occupational impact of the 2003 SARS outbreak in a teaching hospital. CMAJ. 2003;168(10):1245-51.

30. O'Toole MS, Arendt MB, Pedersen CM. Testing an app-assisted treatment for suicide prevention in a randomized controlled trial: effects on suicide risk and depression. Behav Ther. 2019;50(2):421-9.

31. Pellecchia U, Crestani R, Decroo T, Van den Bergh R, Al-Kourdi Y. Social consequences of Ebola containment measures in Liberia. PLoS One. 2015;10(12):e0143036.

32. Recuero R, Gruzd A. Cascatas de Fake News Políticas: um estudo de caso no Twitter. Galáxia (São Paulo). 2019;41(41):31-47.

33. Szklo M. Translational epidemiology: some considerations. Epidemiol Serv Saude. 2015;24(1):161-72. 